SISTEMA
ELETRONIICO
DE REVISTAS
SER I UfPR

\title{
A sustentabilidade em meios de hospedagem: enfoque na materialidade dos serviços
}

\section{Sustainability in the accommodation industry: an approach to the materiality of services}

\author{
Yuli DELLA VOLPI ${ }^{1 *}$, Sônia Regina PAULINO ${ }^{1}$ \\ ${ }^{1}$ Universidade de São Paulo (USP), São Paulo, SP, Brasil. \\ *E-mail de contato: yuli.volpi@usp.br
}

Artigo recebido em 18 de junho de 2018, versão final aceita em 9 de julho de 2019.

RESUMO:

\begin{abstract}
Ao mesmo tempo em que o turismo é reconhecido como atividade causadora de danos ao meio ambiente, a imaterialidade permanece aceita como característica dos serviços em geral, e, portanto, também dos serviços turísticos. O artigo tem como objetivo discutir, à luz de novas abordagens que questionam o entendimento convencional dos serviços, a materialidade dos serviços de hospedagem. Para alcance desse objetivo, é realizada uma pesquisa exploratória. Os dados foram coletados a partir de revisão da literatura sobre desempenho ambiental de meios de hospedagem e sistematizados com base na aplicação do conceito de ciclo de vida (para descrever entradas e saídas associadas aos aspectos ambientais do produto fornecido pelos meios de hospedagem). A estrutura analítica do triângulo dos serviços foi ampliada e utilizada para abordar as fontes de materialidade do serviço. Avançar no entendimento da materialidade dos serviços de hospedagem constitui questão central para o turismo sustentável. Foram identificadas diferentes entradas (energia elétrica, água, alimentos e bebidas, produtos de limpeza, produtos de higiene pessoal, produtos químicos, combustíveis fósseis e outros produtos) e saídas (resíduos orgânicos e inorgânicos, efluentes, emissões atmosféricas, ruídos e odores) associadas às fontes de materialidade dos serviços. Tais entradas e saídas estão fundamentadas no conteúdo material dos serviços de hospedagem, que se manifesta nos sistemas materiais operados (instalações e equipamentos) e nos bens utilizados.
\end{abstract}

Palavras-chave: turismo sustentável; aspectos ambientais; serviço; ciclo de vida.

ABSTRACT: While tourism is recognized as a potential cause of damage to the environment, the immateriality remains as an accepted feature of the services nature, including the tourism services. The article aims to discuss the materiality of accommodation services, in the light of new theoretical approaches that have been challenging 
the conventional understanding of services. In order to do so, an exploratory research is carried out. The data were collected by a literature review on the environmental performance of accommodation businesses, and systematized based on the application of the life cycle concept to describe inputs and outputs associated with the environmental aspects of the product provided by the accommodation businesses. The analytical structure of the services triangle was expanded and used in order to address the sources of the service materiality. Advancing in the understanding of the materiality of accommodation services is a central issue for sustainable tourism. Different inputs (such as energy, water, food and beverages, cleaning products, personal care products, chemicals, fossil fuels, and other products) and outputs (such as organic and inorganic waste, effluents, atmospheric emissions, noise, and odor emissions) associated to the services materiality sources were identified. These inputs and outputs are based on the material content of accommodation services that is manifested in the material systems operated (physical facilities and equipment) and in the goods that are used.

Keywords: sustainable tourism; environmental aspects; service; life cycle concept.

\section{Introdução}

As atividades turísticas trazem uma série de consequências para o destino turístico onde é desenvolvida. Dentre as potenciais consequências positivas que o turismo pode trazer para uma região, destacam-se a geração de empregos, arrecadação de receita, aumento da disponibilidade de recursos para o lazer e o entretenimento, melhora na infraestrutura, promoção de uma melhor imagem local, intercâmbio cultural entre turista e comunidade local e maior consciência sobre a necessidade de proteger o meio ambiente. Já entre as potenciais consequências negativas da atividade turística, pode-se citar o aumento de encargos fiscais e no custo da terra, sazonalidade do emprego, baixos salários, superlotação que degrada o meio ambiente e ameaça à vida selvagem, poluição sonora e do ar, congestionamentos, facilitação do tráfico de drogas e da prostituição, entre outros (Li et al., 2016). Por esse motivo, caso não seja planejado e operacionalizado corretamente, o turismo pode apresentar um grande potencial de degradação dos recursos naturais e dos aspectos socioculturais e econômicos (Peres Jr \& De Rezende, 2011).
Frequentemente, o foco das discussões sobre o turismo está na sua contribuição para a economia. De acordo com a Organização Mundial do Turismo (OMT), por exemplo, o turismo merece particular atenção por ter se tornado um dos setores de crescimento mais rápido do mundo. Ainda segundo a OMT, o turismo representa um fator-chave para o progresso socioeconômico, sendo um dos principais setores do comércio internacional e a principal fonte de renda de alguns países em desenvolvimento (OMT, 2018). Ademais, indo além de sua importância para a economia, o turismo também é reconhecido como uma atividade complexa que abrange recursos sociais, culturais e ambientais, além dos econômicos (Beni, 1990).

Assim, as discussões em torno da sustentabilidade do setor decorrem da constatação de que a atividade não regulamentada, de massa ou alternativa, tem efeitos nocivos para as comunidades locais e para o meio ambiente. É preciso destacar que as empresas de turismo competem pela utilização dos recursos dos locais em que estão inseridas, ao mesmo tempo em que os produtos turísticos dependem da qualidade do meio ambiente (Rattan, 2015). 
Essas discussões relacionadas à busca pela sustentabilidade das atividades do setor de turismo têm ocorrido com mais frequência desde a década de 1990. Vale ressaltar que pesquisadores da área do turismo trabalhavam com questões sociais e ambientais da atividade já nos anos 1970 e 1980. O uso do termo turismo sustentável, porém, só foi incorporado nos anos 1990 (Buckley, 2012).

Embora já tenha sido definido de diferentes formas, o turismo sustentável não corresponde a uma tipologia de turismo, pois toda forma de turismo deveria se esforçar para ser sustentável (PNUMA \& OMT 2005). Assim, a partir do amplo uso do termo turismo sustentável, surgiram também iniciativas que visam estabelecer princípios e critérios que guiem as atividades turísticas rumo à sustentabilidade (UNESCO \& PNUMA, s/d; GSTC, 2018). Em linhas gerais, essas iniciativas estão voltadas para as dimensões ambiental, social, cultural e econômica do turismo.

O setor de turismo é formado por organizações provedoras de serviços, a exemplo de meios de hospedagem, operadoras e agências de viagens. Os meios de hospedagem são definidos como estabelecimentos destinados a prestar serviço temporário de acomodação, assim como outros serviços destinados a satisfazer as necessidades dos hóspedes (BRASIL, 2008). Nesse cenário, os meios de hospedagem representam uma importante atividade de serviço uma vez que, para que seja configurada a atividade turística, é necessário que o viajante pernoite no destino (OMT, 2008). No Brasil, segundo estimativas do Instituto de Pesquisa Econômica Aplicada (IPEA, 2014), os serviços de alojamento estão em segundo lugar na geração de empregos formais no setor de turismo, ficando atrás apenas do número de empregos oferecidos pelo segmento de alimentação.
Assim, os meios de hospedagem representam um segmento que tem recebido bastante atenção nas discussões em torno da sustentabilidade do turismo (Legrand et al., 2013), sendo também contemplados com um conjunto dos Critérios Globais do Turismo Sustentável que apresenta indicadores de desempenho criados especificamente para hotéis (GSTC, 2016).

Porém, ao mesmo tempo em que estudos empíricos mostram as atividades de turismo como potenciais causadoras de impactos negativos ao meio ambiente, persiste na literatura teórica a caracterização dos serviços como imateriais.

Segundo as definiçõoes convencionais, os serviços são bens imateriais e os produtos são tangíveis, commodities físicas. Alguns autores, porém, apresentam a ideia de que esse entendimento tem viés mais casual do que científico, uma vez que a natureza dos produtos imateriais não é desenvolvida e explicada (Hill, 1999). Na verdade, assumiu-se um menor uso de bens de capital, consolidando a ideia da não materialidade dos serviços (Fourcroy et al., 2012).

Essa suposta imaterialidade dos serviços, por sua vez, sustenta o surgimento da ideia de que a economia poderia se tornar "mais verde" a partir da expansão dos serviços como principal setor econômico. Essa visão destaca que o setor de serviços pode contribuir para a melhora na economia a partir da criação de mais empregos - com melhores salários - do que outras atividades, ao mesmo tempo em que não degrada o meio ambiente ou, ao menos, o faz de maneira menos significativa. É a ideia da "desmaterialização da economia" com base no crescimento das atividades de serviços (Young, 2011). Em outras palavras, a característica da imaterialidade atribuída aos serviços está diretamente 
associada ao surgimento da ideia de que a economia poderia se tornar "mais verde" a partir da expansão dos serviços como principal setor econômico em termos de participação no Produto Interno Bruto.

Porém, os avanços no reconhecimento dos serviços como potenciais causadores de impactos negativos ao meio ambiente sinalizam que a concepção de que os serviços são, por natureza, intangíveis não se sustenta. Diante disso, a imaterialidade dos serviços passa a ser tratada como um mito consolidado na literatura por novas abordagens que questionam o entendimento convencional dos serviços (Gadrey, 2000; Gallouj \& Savona, 2009, Djellal \& Gallouj, 2013; Djellal \& Gallouj, 2015a; Fourcroy et al., 2015). No plano teórico, com a tentativa de diferenciar bens e serviços, algumas definições pouco precisas foram se consolidando. De acordo com Fisk et al., (1993), foi a delimitação de quatro características fundamentais que descrevem a natureza dos serviços que forneceu as bases para a distinção entre estes e os bens. Essas características são: a intangibilidade, a inseparabilidade, a heterogeneidade e a perecibilidade. A inseparabilidade é conferida pela simultaneidade da produção e do consumo; a heterogeneidade pela não padronização; e a perecibilidade deve-se à impossibilidade de se realizar um inventário (Lovelock \& Gummesson, 2004). Observa-se, também, que a questão da intangibilidade aparece relacionada com a da perecibilidade, já que se considera que os serviços não podem ser estocados e são consumidos no momento da produção (Kon, 2004). Os serviços são apontados como bens imateriais enquanto os produtos são tangíveis. Porém, esse entendimento parece ter viés mais casual do que científico, uma vez que a natureza dos produtos imateriais não é desenvolvida e explicada (Hill, 1999).
Essas novas abordagens mostram que o setor de serviços tem sido, durante muito tempo, alvo de diversas concepções errôneas, resultantes de um processo de definição que incluiu no mesmo grupo todas as atividades que não podiam ser encaixadas no primeiro ou no segundo setores da economia (Gummesson, 2010). Como resultado, uma das ideias mais difundidas de diferenciação entre bens e serviços foi aquela que discorre sobre a característica intangível (ou imaterial) dos serviços.

Foi a partir dos anos 2000 que o entendimento da natureza dos serviços passou a ser alvo de questionamentos mais frequentes. Segundo Gadrey (2002), nenhuma das características tradicionalmente designadas aos serviços representa a realidade ou é suficiente para permitir um progresso em torno da definição e da avaliação do resultado ou produto dos serviços. Além disso, pesquisas apontam que o entendimento dos serviços como imateriais tem levado a falhas no momento de avaliar a performance ambiental do setor (Gallouj \& Savona, 2009; Fourcroy et al., 2012). Deve-se levar em consideração que todos os processos produtivos, incluindo a produção de serviços, transformam combinações de energia, material e informação em combinações novas e mais valorizadas (Tether \& Metcalfe, 2004).

Em suma, a concepção da imaterialidade dos serviços tem sido contestada em novas abordagens da economia de serviços, e essa contestação está diretamente relacionada ao fato de que os serviços têm sido reconhecidos como consumidores significativos de recursos naturais, algo que, por vezes, é apenas parcialmente considerado nas estatísticas relacionadas ao desempenho ambiental do setor (Fourcroy et al., 2012; Djellal \& Gallouj, 2015b). Assim sendo, os serviços são imateriais ao não produzirem, como resultado final, bens tangíveis. 
Mas, ao mesmo tempo, possuem diferentes fontes de materialidade, de acordo com o tipo de serviço, para que possam ocorrer enquanto processo produtivo (Djellal \& Gallouj, 2016). Em outras palavras, o produto e o processo produtivo do serviço são entendidos como indissociáveis.

Isso posto, o artigo busca abordar uma questão fundamental para a análise da sustentabilidade no turismo: como se configuram as fontes de materialidade dos serviços? O recorte da pesquisa recai sobre os meios de hospedagem, considerando os requisitos ambientais do turismo sustentável. Assim, a pesquisa de caráter exploratório tem como objetivo discutir, à luz de novas abordagens que questionam o entendimento convencional dos serviços, a materialidade dos serviços de hospedagem. Os dados foram coletados a partir de revisão da literatura sobre desempenho ambiental de meios de hospedagem e sistematizados com base na aplicação do conceito de ciclo de vida (para descrever entradas e saídas associadas aos aspectos ambientais do produto fornecido pelos meios de hospedagem). E foi ampliada e utilizada a estrutura analítica do triângulo dos serviços para abordar as fontes de materialidade do serviço.

Após esta introdução, é feita uma contextualização sobre o turismo sustentável e os meios de hospedagem. Nas seções seguintes são apresentados a metodologia, os resultados da pesquisa e as considerações finais.

\section{Turismo sustentável e os meios de hospedagem}

Durante muito tempo, as discussões em torno do turismo estiveram focadas quase que exclusiva- mente em seus aspectos econômicos e no papel do turismo para a evolução do Produto Interno Bruto (PIB) (Körössy, 2008). O Conselho Mundial de Viagens e Turismo (WTTC), por exemplo, produz relatórios e previsões anuais sobre os impactos econômicos do setor, para 185 países, que deixam evidente a importância do turismo para a economia. Em 2016, o crescimento do setor de viagens e turismo superou o crescimento da economia global pelo sexto ano consecutivo, passando a representar $10,2 \%$ do Produto Interno Bruto (PIB) mundial e contribuindo para a existência de 292 milhões de empregos (aproximadamente um para cada dez postos de trabalho no mundo). No Brasil, estima-se que o setor de viagens e turismo contribui para 7,8\% do total de empregos (incluindo empregos gerados indiretamente), e o país aparece em $11^{\circ}$ entre as nações em que o setor apresenta maior contribuição para o PIB, em termos absolutos (WTTC, 2017).

Aos poucos, porém, o turismo também tem sido associado a questões ambientais e sociais, o que tem motivado diversos destinos a buscar por um turismo sustentável (Körössy, 2008). Afinal, é reconhecido que o turismo pode se tornar um motor para a degradação, caso não conte com planejamento e gestão eficazes (OMT, 2004).

De acordo com uma definição geral de turismo sustentável indicada pela OMT em 1998, o desenvolvimento do turismo sustentável deve satisfazer as necessidades dos turistas e dos destinos turísticos enquanto assegura as oportunidades futuras, a partir de uma gestão de recursos que satisfaça as necessidades econômicas, sociais e estéticas e mantenha a integridade cultural, os processos ecológicos, a diversidade biológica e os sistemas de suporte à vida (UNESCO \& PNUMA, s/d). Já no ano 2000, durante o Primeiro Workshop Internacional sobre 
Certificações em Ecoturismo e Turismo Sustentável, em Nova Iorque, o turismo sustentável foi definido, no Acordo Mohonk, como aquele que procura minimizar impactos socioculturais e ambientais ao mesmo tempo em que proporciona benefícios econômicos para as comunidades e países em que se insere, sendo, portanto, distinto do ecoturismo, que se refere ao turismo sustentável que possui foco nas áreas naturais e beneficia o ambiente e as comunidades, promovendo conscientização e apreciação ambiental e cultural (Mohonk Agreement, 2000). Cinco anos depois, um documento desenvolvido pela OMT e pelo PNUMA (PNUMA \& OMT, 2005) estabeleceu que o turismo sustentável não corresponde a uma tipologia de turismo, pois toda forma de turismo deveria se esforçar para ser sustentável, sendo essa uma atividade capaz de beneficiar socialmente e economicamente as comunidades locais, além de conscientizar e apoiar a conservação ambiental.

Cada vez mais, estudos empíricos acerca dos impactos e do desempenho ambiental do turismo vêm sendo amplamente desenvolvidos, destacando práticas de gestão e apresentando um enfoque que contribui para avançar na investigação da sustentabilidade no setor. Afinal, o turismo sustentável envolve a compreensão dos impactos causados pela atividade (Beni, 2003). Sealey \& Smith (2014), por exemplo, mostram que um resort pode produzir $35 \%$ do total de resíduos sólidos gerados em uma ilha das Bahamas, enquanto Felix \& Santos (2013) propõem uma metodologia de avaliação de desempenho ambiental para o setor hoteleiro. Assim, a questão ambiental deve continuar recebendo cada vez mais atenção, especialmente em destinações de equilíbrio ambiental delicado, conforme se adquirem maiores informações sobre os possíveis impactos negativos gerados pelos estabelecimentos que compõem o setor.

Verifica-se, ainda, que a promoção da sustentabilidade no turismo demanda diferentes stakeholders, envolvendo turistas, organizações não governamentais (ONGs), empresas, governo etc. (Lee \& Hsieh, 2016). Diante disso, surgem cada vez mais iniciativas - como o estabelecimento de princípios, critérios e padrões de certificação - que buscam promover o desenvolvimento da atividade turística de forma a mitigar seus potenciais impactos negativos. Em âmbito internacional, uma publicação da UNESCO e do PNUMA traz uma síntese de diferentes declarações de princípios criados para o turismo sustentável. Segundo o documento (UNESCO \& PNUMA, s/d), o turismo sustentável tem como princípios básicos: 1) Melhorar o bem-estar das comunidades; ou seja, garantir o bem-estar econômico, social e cultural das comunidades em que o turismo se insere; 2) Apoiar a proteção do ambiente natural e cultural; pois o turismo sustentável deve permitir o uso de recursos naturais e culturais para a obtenção de lucro econômico ao mesmo tempo em que garante que esses recursos (culturais e naturais) não sejam deteriorados, além de impulsionar a criação ou fortalecer a proteção da natureza e a manutenção de valores culturais; 3 ) Reconhecer a qualidade do produto e a satisfação do turista; já que a qualidade dos produtos turísti$\cos$ - que não se caracterizam apenas por critérios materiais (transporte, alojamento, alimentação) mas também por critérios não materiais (hospitalidade, experiências) - é uma questão chave para seu sucesso econômico; 4) Aplicar gerenciamento e monitoramento adaptativo; ou seja, buscar uma gestão e monitoramento que segue os princípios básicos de uso sustentável dos recursos, a fim de 
garantir que o turismo seja desenvolvido de forma ecológica, econômica e socialmente sustentável. No Brasil, os princípios desenvolvidos em junho de 2002, no âmbito do Conselho Brasileiro de Turismo Sustentável(CBTS) em conjunto com entidades ambientalistas, empresários, especialistas em turismo e representantes de movimentos sociais, podem ser usados como referência nacional (MTUR, 2007).
Já com relação ao desenvolvimento de critérios para o turismo sustentável, destaca-se a formulação de conjuntos de Critérios Globais do Turismo Sustentável, criados para destinos turísticos, hotéis e operadoras de turismo (GSTC, 2016). Desde a publicação dos critérios desenvolvidos para hotéis e operadoras de turismo, em 2008, esses conjuntos já passaram por três revisões que procuraram

QUADRO 1 - Critérios Globais do Turismo Sustentável para Hotéis.

\begin{tabular}{|c|c|}
\hline $\begin{array}{l}\text { A) Planejamento eficaz para a sustentabilidade } \\
\text { A.1. Implementação de Sistema de Gestão da Sustentabilidade } \\
\text { A.2. Conformidade legal } \\
\text { A.3. Comunicação de política de sustentabilidade, ações e de- } \\
\text { sempenho } \\
\text { A.4. Compromisso de equipe } \\
\text { A.5. Experiência do consumidor } \\
\text { A.6. Promoção correta da empresa } \\
\text { A.7. Projeto, construção de edifícios e infraestrutura que este- } \\
\text { jam de acordo com requisitos locais de zoneamento; respeitem } \\
\text { o patrimônio natural e cultural; utilizem princípios localmente } \\
\text { apropriados de construção sustentável e ofereçam acesso a pes- } \\
\text { soas com necessidades especiais } \\
\text { A.8. Direito de propriedade legal e de acordo com os direitos } \\
\text { indígenas/comunidade local } \\
\text { A.9. Oferecimento de informação sobre os arredores e sobre o } \\
\text { comportamento adequado para o turista } \\
\text { A.10. Envolvimento no planejamento e gestão do turismo sus- } \\
\text { tentável da região }\end{array}$ & $\begin{array}{l}\text { B) Maximização de beneficios sociais e econômicos para a comunidade local } \\
\text { B.1. Apoio à comunidade } \\
\text { B.2. Oferta de emprego para a comunidade } \\
\text { B.3. Aquisição de bens e serviços locais } \\
\text { B.4. Apoio aos pequenos empresários locais } \\
\text { B.5. Políticas contra qualquer forma de exploração (comercial, sexual } \\
\text { etc.) } \\
\text { B.6. Oferecimento de oportunidades de emprego igualitárias para as mi- } \\
\text { norias } \\
\text { B.7. Condições decentes de trabalho } \\
\text { B.8. Atividades que não comprometam a prestação de serviços básicos } \\
\text { para a comunidade (água, energia, alimento etc.) } \\
\text { B.9. Atividades que não afetem negativamente o acesso local a meios } \\
\text { de subsistência (habitação, transporte, terra, recursos de água doce etc.) }\end{array}$ \\
\hline $\begin{array}{l}\text { C) Melhoramento do patrimônio cultural } \\
\text { C.1. Respeito a acordos de boas práticas para visitas a comu- } \\
\text { nidades indígenas e locais cultural ou historicamente sensíveis } \\
\text { C.2. Proteção do patrimônio cultural sem impedir o acesso de } \\
\text { residentes locais } \\
\text { C.3. Valorização e incorporação de elementos de cultura local } \\
\text { tradicional e contemporânea, com respeito aos direitos de pro- } \\
\text { priedade intelectual } \\
\text { C.4. Não há venda ilegal de artefatos históricos ou arqueológicos }\end{array}$ & $\begin{array}{l}\text { D) Redução dos impactos negativos no ambiente } \\
\text { D.1. Conservação dos recursos (preferência por fornecedores ambiental- } \\
\text { mente sustentáveis; redução de resíduos; diminuição no consumo de água } \\
\text { e energia; fomento a energias renováveis) } \\
\text { D.2. Redução da poluição (controle das emissões de gases de efeito es- } \\
\text { tufa, tratamento e reuso de águas residuais, plano de manejo de resíduos } \\
\text { sólidos orgânicos e inorgânicos; minimização no uso de substâncias peri- } \\
\text { gosas; minimização de poluição sonora, visual, do ar e do solo) } \\
\text { D.3. Conservação da biodiversidade, ecossistemas e paisagens (espécies } \\
\text { silvestres não são ilegalmente vendidas, consumidas ou exibidas; animais } \\
\text { silvestres não são mantidos em cativeiro sem regulação; são tomadas me- } \\
\text { didas para evitar a introdução de espécies exóticas invasoras; a empresa } \\
\text { apoia ações de conservação, áreas protegidas etc.; interações com a vida } \\
\text { selvagem são geridas de maneira responsável e não são invasivas; a visi- } \\
\text { tação segue regras apropriadas) }\end{array}$ \\
\hline
\end{tabular}

FONTE: baseado em GSTC (2016) e Rainforest Alliance (s/d). 
considerar os comentários e sugestões recebidos, encontrando-se constantemente em processo de atualização. Ademais, os Critérios Globais do Turismo Sustentável (GSTC Criteria) voltados para hotéis e operadoras de turismo encontram-se organizados em torno de quatro temas principais: planejamento eficaz para a sustentabilidade, maximização de benefícios sociais e econômicos para a comunidade local, melhoramento do patrimônio cultural e redução de impactos negativos no ambiente. Os Critérios Globais do Turismo Sustentável para hotéis, separados de acordo com seu tema geral, podem ser visualizados no Quadro 1, elaborado com base em GSTC (2016) e Rainforest Alliance (s/d).

Verifica-se que os meios de hospedagem representam um segmento que tem recebido bastante atenção nas discussões em torno da sustentabilidade no turismo com o surgimento de diversos padrões que estabelecem requisitos de sustentabilidade para meios de hospedagem, como, por exemplo: Green Globe, Green Key, Eco Hotels Certified (ehc) e HI Quality and Sustainability. No Brasil, já existe uma certificação específica para meios de hospedagem, a NBR 15401 Meios de Hospedagem - Sistema de Gestão da Sustentabilidade. Assim como ocorre com a definição de princípios e critérios para o turismo sustentável, os padrões de certificação adotam, de modo geral, requisitos relacionados às dimensões ambiental, social, cultural e econômica do turismo sustentável. Os requisitos ambientais para os meios de hospedagem englobam: conformidade com a legislação ambiental, eficiência energética, gestão de resíduos e efluentes, redução no consumo de água, controle do uso de produtos químicos, seleção de insumos, minimização de impactos nos ecossistemas naturais, conservação de áreas naturais e redução da poluição atmosférica.

\section{Metodologia}

Trata-se de uma pesquisa exploratória baseada em revisão da literatura com a coleta de dados qualitativos, posteriormente sistematizados a partir da aplicação do enfoque do conceito de ciclo de vida do produto e analisados com base no modelo do triângulo dos serviços. As pesquisas exploratórias têm como objetivo desenvolver, esclarecer e modificar conceitos e ideias (Gil, 1991). A seguir, são explicadas detalhadamente as etapas realizadas para levantamento, sistematização e análise dos dados.

\subsection{Revisão da literatura}

A coleta de dados, relacionados aos aspectos ambientais do produto fornecido pelos meios de hospedagem, foi feita nas bases Scopus e Scielo e finalizada em janeiro de 2017. No primeiro grupo de palavras-chave, procurou-se contemplar diferentes abordagens de questões ambientais utilizadas em estudos sobre meios de hospedagem. Assim, as palavras selecionadas foram: Gestão ambiental (ou Environmental management, para pesquisa em inglês); Impacto ambiental (ou Environmental impact, para pesquisa em inglês); Desempenho ambiental (ou Environmental performance, para pesquisa em inglês); Aspectos ambientais (ou Environmental aspect, para pesquisa em inglês). O segundo grupo de palavras-chave foi formado com o intuito de englobar diversas categorias de meios de hospedagem. As palavras utilizadas foram: Hotel; Cama e café (ou Bed and breakfast, para pesquisa em inglês); Hostel; Resort; Pousada (ou Inn, para pesquisa em inglês); Hospedagem (ou Accommodation, para pesquisa em inglês); Alojamento (ou Lodg*, para 
pesquisa em inglês, visando contemplar variações da palavra como lodging e lodge).

A combinação de palavras-chave foi usada na base de dados SCOPUS, a partir do uso dos filtros: ("Environmental management" OR "Environmental impact" OR "Environmental performance" OR "Environmental aspects") AND (Hotel OR "Bed and breakfast" OR Hostel OR Resort OR Inn OR accommodation OR lodg*), para título do artigo, resumo e palavras-chave. Foram considerados artigos e revisões, nas línguas portuguesa e inglesa, publicados em revistas científicas (journals) no período de 2000 a 2017, das áreas de ciências ambientais e ciências sociais aplicadas, selecionando inicialmente 345 artigos. Esses artigos foram analisados a partir da leitura do título e do resumo de cada um buscando identificar as publicações com foco na análise dos meios de hospedagem. Com base nesses procedimentos, 70 artigos foram selecionados e lidos integralmente, tendo sido identificados dados relacionados aos aspectos ambientais dos serviços de hospedagem em 65 artigos. Os mesmos procedimentos foram utilizados para a busca na base de dados SCIELO, a partir dos filtros: ("gestao ambiental" OR "impacto ambiental" OR "desempenho ambiental" OR "aspectos ambientais") AND (Hotel OR "cama e cafe" OR Hostel OR Resort OR pousada OR hospedagem OR alojamento), utilizando os campos de pesquisa "integrada" e "regional". Devido à quantidade reduzida de artigos selecionados nessa base de dados em que puderam ser encontrados dados relacionados aos aspectos ambientais dos serviços de hospedagem, também foi realizada uma pesquisa complementar na base SCIELO, aplicando-se os filtros: (turismo OR hotel) AND (sustentabilidade OR ambiental) e utilizando os campos de pesquisa "integrada" e "regional".
Assim, as pesquisas na base de dados SCIELO resultaram juntas em 08 artigos utilizados neste estudo. Desse modo, o total de artigos considerados nesta pesquisa foi de 73 publicações que forneceram dados sobre o fenômeno investigado.

\subsection{Aplicação do enfoque do conceito de ciclo de vida}

Utilizou-se o enfoque do conceito de ciclo de vida proposto no Guia para consideração de questões ambientais em normas de produtos (ABNT ISO Guia 64, 2010) a fim de refinar a classificação dos aspectos ambientais como entradas ou saídas relacionadas ao ciclo de vida do produto fornecido pelos meios de hospedagem e que são geradas nos estágios de produção e uso dos serviços de hospedagem. O conceito de ciclo de vida, apresentado na figura 1 , refere-se à consideração de todos os aspectos ambientais de um produto em todos os estágios de seu ciclo de vida, apontando como estágios principais: a aquisição de materiais, a produção, o uso e o fim de vida do produto (bem e serviço).

Os aspectos ambientais são definidos como qualquer componente do produto que possa interagir com o meio ambiente durante seu tempo de vida. E devem ser identificados levando em conta as entradas e saídas. As entradas presentes durante o ciclo de vida de um produto se referem ao consumo de recursos necessários nos diferentes estágios desse ciclo de vida, enquanto que as saídas compreendem produtos intermediários ou coprodutos, emissões atmosféricas, descargas na água e no solo, resíduos sólidos e outros lançamentos. O conceito do ciclo de vida do produto também pode ser usado para abordar atividades de serviço 
(ABNT ISO Guia 64, 2010). O enfoque do ciclo de vida do produto já foi utilizado em pesquisas sobre o setor de turismo, mostrando-se útil para o entendimento da evolução dos produtos turísticos e destinações (Moss et al., 2003; Weiermair et al., 2007). Nessa pesquisa, propõe-se que o conceito pode contribuir para análise das fontes de materialidade do serviço. Dessa forma, a interpretação dos aspectos ambientais presentes no ciclo de vida dos serviços de hospedagem é realizada com base em abordagens teóricas da materialidade por meio da estrutura analítica do triângulo dos serviços.

\subsection{Aplicação do triângulo dos serviços}

Por fim, a pesquisa utilizou a estrutura analítica do triângulo dos serviços, proposta por Gadrey (2002), para analisar os serviços de hospedagem. A definição de serviço que embasa essa estrutura é:

Uma atividade de serviço é uma operação destinada a trazer uma mudança de estado em uma realidade $\mathrm{C}$, que é de propriedade ou utilizada por consumidores $\mathrm{B}$, sendo a mudança efetuada por um prestador de serviços A a pedido de B e, em muitos casos, em

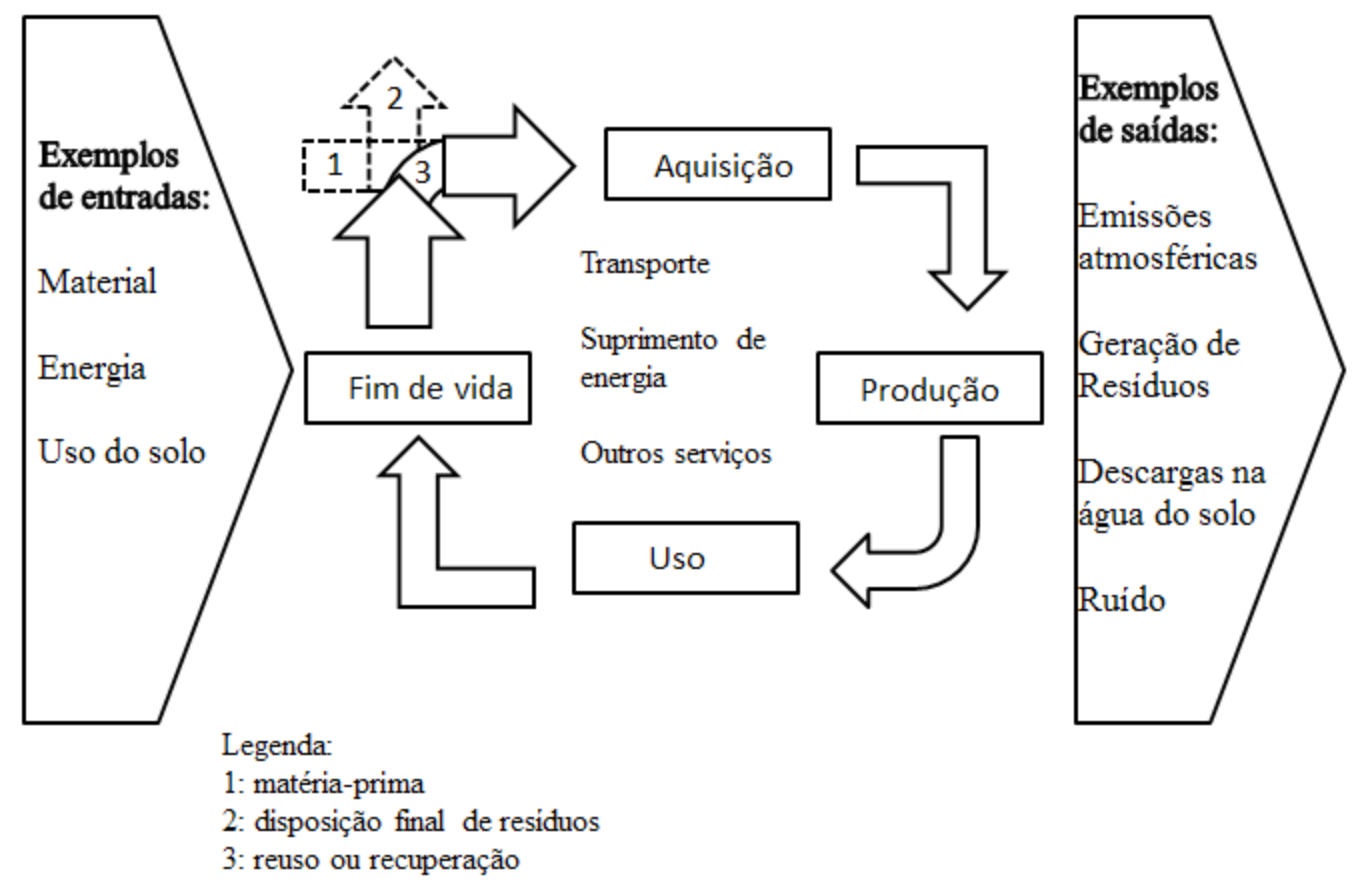

FIGURA 1 - Enfoque do ciclo de vida do produto.

FONTE: ABNT ISO Guia 64 (2010). 
colaboração com ele/ela, mas sem levar à produção de um bem que pode circular na economia independentemente do meio C (Gadrey, 2000, p. 375, tradução livre).

Por se tratar de uma representação sob a forma de um triângulo $\mathrm{ABC}$, essa definição pode ser visualizada por meio da Figura 2.

Buscou-se, assim, trazer essa abordagem da economia de serviços para esclarecer as fontes de materialidade dos serviços de hospedagem.

\section{As fontes de materialidade dos serviços de hospedagem}

Um total de 6.021 meios de hospedagem foi objeto de estudo das publicações consideradas com base em revisão da literatura (excluindo-se os trabalhos que objetivavam propor a criação de modelos de empreendimentos, não focando casos concretos). A distribuição desses meios de hospedagem, de acordo com sua região de localização, pode ser observada na Figura 3.

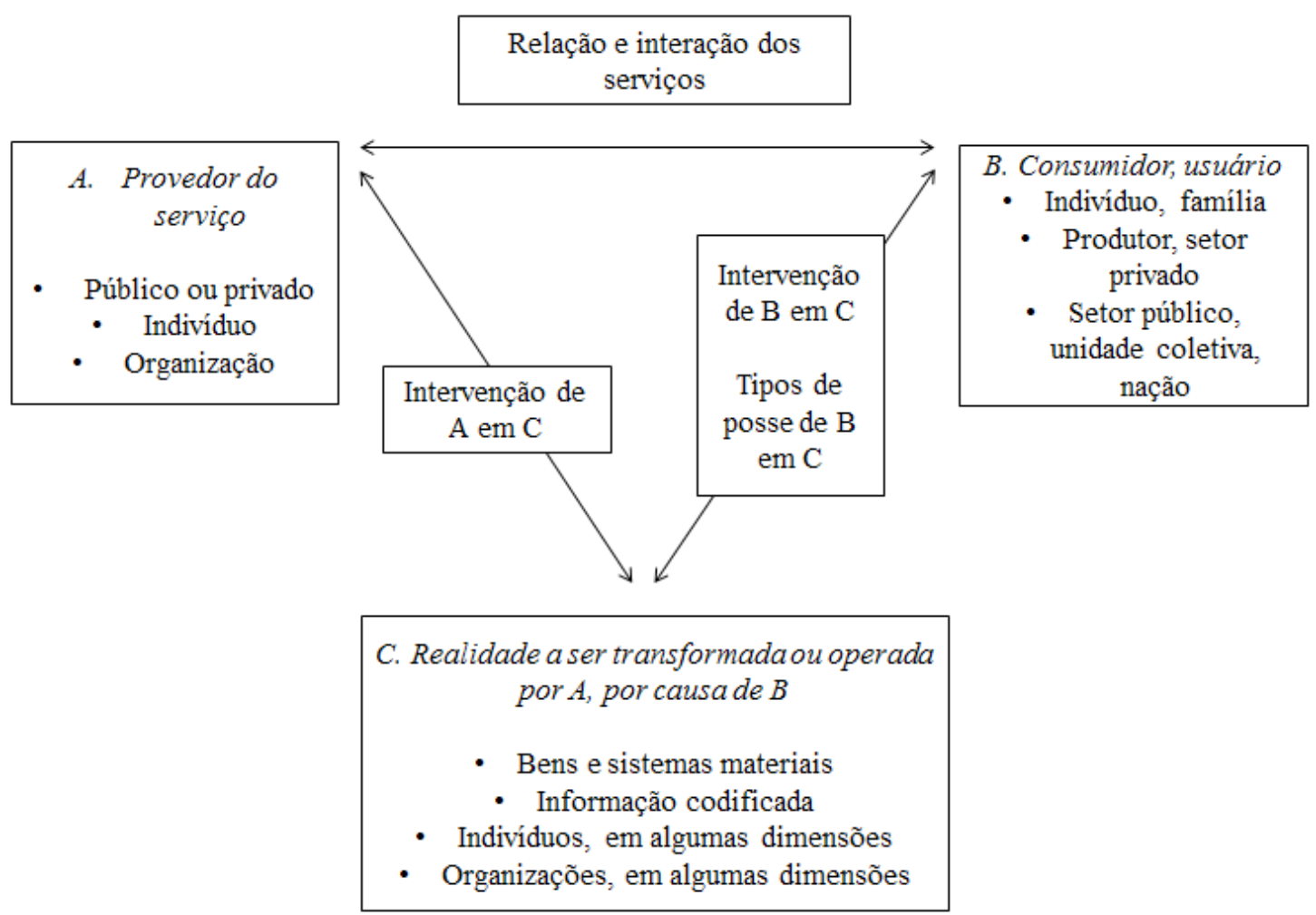

FIGURA 2 - Triângulo dos serviços.

FONTE: Gadrey (2002, p. 42, tradução livre). 


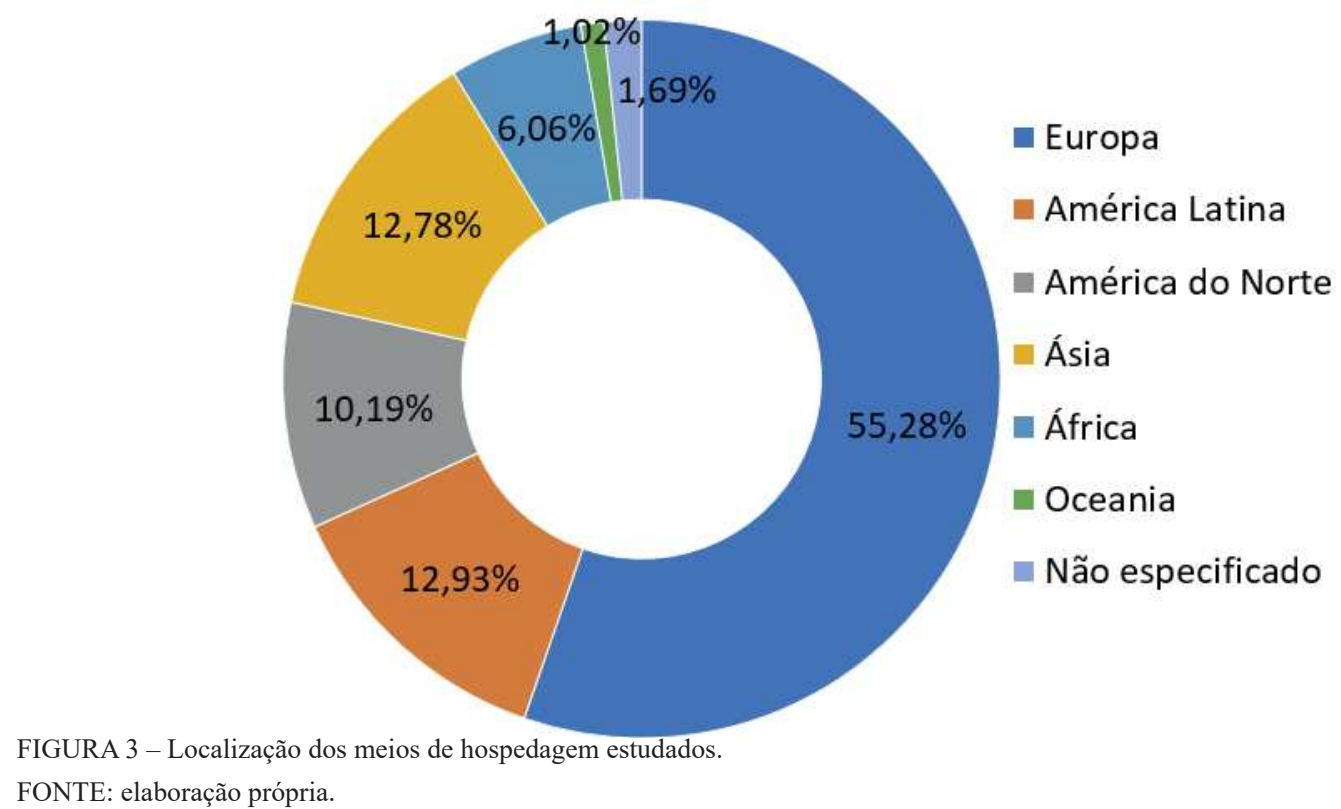

FONTE: elaboração própria.

A legenda "não especificado", na Figura 3, refere-se a um estudo que enviou questionários para diversas cadeias de hotéis, sem mencionar de que países e cadeias foram obtidas respostas. Pode-se notar que a maior parte dos meios de hospedagem estudados é localizada na Europa, região em que tem se destacado o desenvolvimento de certificações do turismo sustentável.

Ademais, se faz importante mostrar a diferença entre as categorias de meios de hospedagem consideradas nas publicações. A maioria dos estabelecimentos, aproximadamente $80 \%$, tratava-se de hotéis, como pode ser observado na Figura 4. É importante ressaltar que a classificação dos meios de hospedagem pode variar de acordo com o país em que estão inseridos. Sendo assim, foram consi- deradas as classificações adotadas pelos autores das publicações. Na Figura 4, a legenda "não especificado" se refere ao número de meios de hospedagem que não tiveram sua categoria explicitada nos artigos. Já a legenda "outros meios de hospedagem" engloba resorts, ski resorts, pousadas, campings, apart-hotéis e similares.

A partir da revisão da literatura, foi realizada a descrição dos aspectos ambientais associados às atividades desenvolvidas nas fases de produção e uso de serviços de hospedagem (Tabela 1).

Os aspectos ambientais são classificados em entradas e saídas relevantes geradas ao longo dos estágios de produção e uso dos serviços de hospedagem, tendo como base o enfoque do conceito do ciclo de vida (ABNT ISO Guia 64, 2010). As entra- 
das presentes durante o ciclo de vida de um produto se referem ao consumo de recursos necessários nos diferentes estágios desse ciclo de vida, e as saídas compreendem produtos intermediários ou coprodutos, emissões atmosféricas, descargas na água e no solo, resíduos sólidos e outros lançamentos. Conforme mostrado na Figura 5, as entradas presentes nos estágios de produção e uso dos serviços de hospedagem são: energia elétrica, água, alimentos e bebidas, produtos de limpeza, produtos de higiene pessoal, produtos químicos, combustíveis fósseis e outros produtos. Já as saídas se referem a resíduos orgânicos e inorgânicos, efluentes, emissões atmosféricas, ruídos e odores.

A aplicação do triângulo dos serviços - adaptado para a produção e o uso dos serviços de hospedagem e ampliado para a incorporação dos aspectos ambientais associados às fontes de materialidade do serviço, na Figura 6, - também permite que sejam consideradas as prestações elementares de intervenção e preparação.

Todos os processos produtivos transformam combinações de energia, material e informação em novas combinações mais valorizadas (Tether \& Metcalfe, 2004). Como apontado por Gadrey (2010), a produção e o consumo dos serviços podem causar tantos danos ao meio ambiente quanto os demais produtos. Nesse sentido amplo, a prestação de um serviço é entendida como resultado da combinação, em proporções variáveis, de três prestações elementares (intervenção, preparação e deslocamento) nas quais são identificadas fontes de materialidade dos serviços (Djellal \& Gallouj, 2016; Fourcroy et al, 2015).

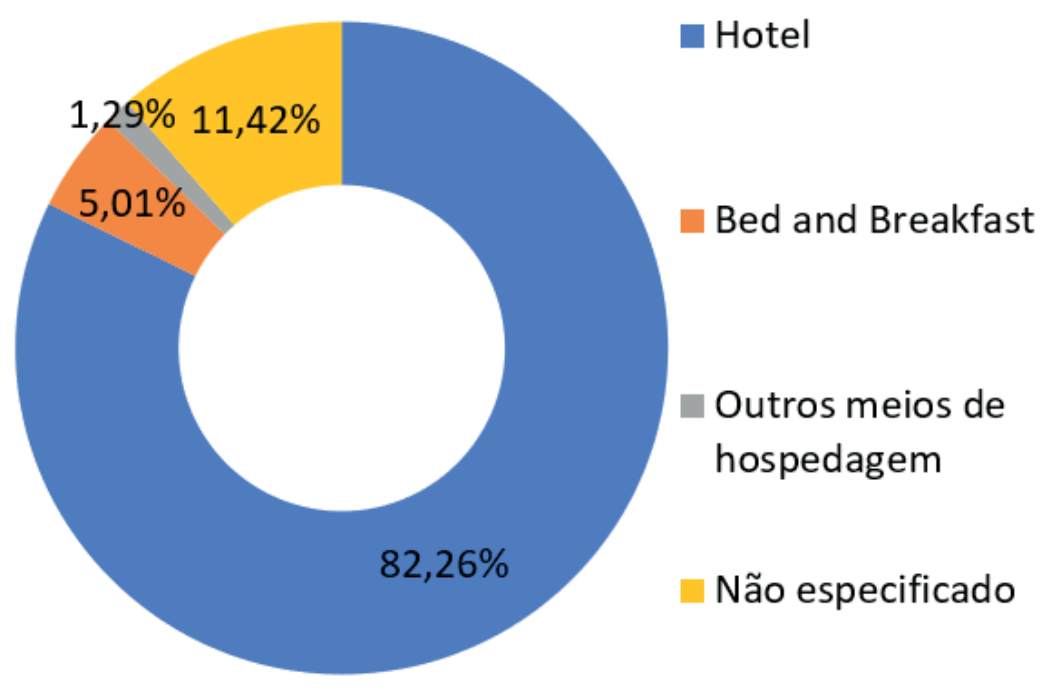

FIGURA 4 - Distribuição dos meios de hospedagem segundo categoria.

FONTE: elaboração própria. 
TABELA 1 - Aspectos ambientais de serviços de hospedagem.

\section{Aspectos ambientais e descrição \\ Fontes}

Alonso-Almeida et al. (2017), Alonso-Almeida \& Rodríguez-Antón (2011), Armas-Cruz (2011), Ayuso (2006), Bagur-Femenias et al. (2016), Bardhan (2012), Best \& Thapa (2013), Boemi \& Avdimiotis (2015), Carrenho et al. (2012), Chan (2011), Chan et al. (2009), Chan \& Wong (2006), Chen \& Huang (2015), Chou (2014), Chung \& Parker (2008), Cortijo (2003).Cumo et al. (2015), Torres et al. (2016), Dief \& Font (2012), Dodds \& Holmes

\section{Consumo de energia:}

Consumo de eletricidade proveniente de fornecedor externo ou gerada no próprio estabelecimento, usada nos equipamentos elétricos e eletrônicos, na iluminação e na climatização dos meios de hospedagem e energia térmica gerada no próprio meio de hospedagem para uso em cocção, nos sistemas de aquecimento central e sistemas de aquecimento da água

\section{Consumo de água:}

Consumo de água nas piscinas e nos equipamentos presentes nos banheiros e lavanderias (2011), Duglio \& Beltramo (2016), Echarri et al. (2017), Fazelpour et al. (2014), Filimonau et al. (2011), Font et al. (2012), Fraj et al. (2015), Freitas \& Almeida (2010), Garcia-Pozo et al. (2014), Georgei \& Bombeck (2012), Graci \& Dodds (2008), Hu et al. (2015), Karatzoglou \& Spilanis (2010), Kasim \& Dzakiria (2009), Lai et al (2012), Le et al. (2006), Legrand et al. (2012), Leonidou et al. (2015), López-Gamero et al. (2011), Medeiros et al. (2012), Mensah (2014a, 2014b), Mishra (2016), Molina-Azorin (2015), Molina-Azorín et al. (2009), Oreja-Rodríguez \& Armas-Cruz (2012), Park \& Kim (2014), Pereira-Moliner et al. (2012), Pistorello et al. (2015), Qi et al. (2017), Rabontu \& Babucea (2015), Reynolds (2013), Rivera (2004, 2002), Rivera \& De Leon (2005), Salgado \& Colombo (2015), Sánchez-Medina et al. (2016), Sanchez-Ollero et al. (2012), Santos et al. (2017), Shah (2011), Sharma (2009), Sousa \& Eusébio (2013), Sozer (2010), Styles et al. (2015), Tang et al. (2014), Trung \& Kumar (2005), Warnken et al. (2005, 2004), Xuchão et al. (2010), Zubair et al (2011)

Alonso-Almeida et al. (2017), Alonso-Almeida \& Rodríguez-Antón (2011), Armas-Cruz (2011), Ayuso (2006), Bagur-Femenias et al. (2016), Bardhan (2012), Best \& Thapa (2013), Boemi \& Avdimiotis (2015), Carrenho et al. (2012), Chan (2011), Chan \& Wong (2006), Chan et al. (2009), Chou (2014), Chung \& Parker (2008), Cortijo (2003), Dief \& Font (2012), Dodds \& Holmes (2011), Duglio \& Beltramo (2016), Filimonau et al. (2011), Font et al. (2012), Fraj et al. (2015), Freitas \& Almeida (2010), Garcia-Pozo et al. (2014), Georgei \& Bombeck (2012), Graci \& Dodds (2008), Hu et al. (2015), Karatzoglou \& Spilanis (2010), Kasim \& Dzakiria (2009), Lai et al (2012), Le et al. (2006), Legrand et al. (2012), Leonidou et al. (2015), López-Gamero et al. (2011), Medeiros et al. (2012), Mensah (2014a, 2014b), Mishra (2016), Molina-Azorin (2015), Molina-Azorín et al. (2009), Oreja-Rodríguez \& Armas-Cruz (2012), Park \& Kim (2014), Pereira-Moliner et al. (2012), Pistorello et al. (2015), Qi et al. (2017), Rabontu \& Babucea (2015), Reynolds (2013), Rivera (2004, 2002), Rivera \& De Leon (2005), Salgado \& Colombo (2015), Sánchez-Medina et al. (2016), Sanchez-Ollero et al. (2012), Santos et al. (2017), Shah (2011), Sharma (2009), Sousa \& Eusébio (2013), Sousa et al. (2016), Sozer (2010), Styles et al. (2015), Tang et al. (2014), Tortella \& Tirado (2011), Trung \& Kumar (2005), Warnken et al. (2005, 2004), Xuchão et al. (2010), Zubair et al (2011).

Alonso-Almeida et al. (2017), Armas-Cruz (2011), Chan et al. (2009), Chen \& Huang (2015), Chou (2014), Dief \& Font (2012), Dodds \& Holmes

\section{Consumo de alimentos/bebidas:} (2011), Filimonau et al. (2011), Font et al. (2012), Graci \& Dodds (2008), Hu et al. (2015), Karatzoglou \& Spilanis (2010), Leonidou et al. (2015), Mishra (2016), Oreja-Rodríguez \& Armas-Cruz (2012), Park \& Kim (2014), Pistorello et al. (2015), Reynolds (2013), Rivera (2002), Salgado \& Colombo (2015), Sousa \& Eusébio (2013), Tortella \& Tirado (2011), Trung \& Kumar (2005). 


\section{Consumo de produtos de limpeza:}

Detergentes e demais produtos usados na manutenção da limpeza do meio de hospedagem e na lavagem da roupa de cama e banho

\section{Consumo de produtos de higiene pessoal e cosméticos:}

Oferecimento de sabonetes e shampoos
Alonso-Almeida et al. (2017), Alonso-Almeida \& Rodríguez-Antón (2011), Bagur-Femenias et al. (2016), Dodds \& Holmes (2011), Filimonau et al. (2011), Freitas \& Almeida (2010), Mensah (2014a), Mishra (2016), Rivera (2002), Salgado \& Colombo (2015), Sousa \& Eusébio (2013), Styles et al. (2015), Tang et al. (2014).

Consumo de produtos químicos:

Uso de agrotóxicos para jardinagem e tratamento de campos de golfe e uso de cloro nas piscinas

\section{Consumo de combustíveis fósseis:}

Gás e diesel utilizados para a geração de energia térmica e elétrica no próprio meio de hospedagem

\section{Consumo de outros produtos:}

Papéis, embalagens, baterias, pilhas
Alonso-Almeida et al. (2017), Bagur-Femenias et al. (2016), Filimonau et al. (2011), Graci \& Dodds (2008), Hu et al. (2015), Leonidou et al. (2015), López-Gamero et al. (2011), Rabontu \& Babucea (2015), Rivera (2002), Sousa \& Eusébio (2013), Styles et al. (2015), Trung \& Kumar (2005).

Cortijo (2003), López-Gamero et al. (2011), Sousa \& Eusébio (2013).

Bardhan (2012), Chan (2011), Chen \& Huang (2015), Filimonau et al. (2011), Georgei \& Bombeck (2012), Hu et al. (2015), Kasim \& Dzakiria (2009), Lai et al (2012), Mishra (2016), Molina-Azorín et al. (2009), Qi et al. (2017), Rabontu \& Babucea (2015), Sharma (2009), Sozer (2010), Styles et al. (2015), Tang et al. (2014), Trung \& Kumar (2005), Warnken et al. (2004, 2005),Xuchão et al. (2010), Zubair et al (2011).

Alonso-Almeida et al. (2017), Alonso-Almeida \& Rodríguez-Antón (2011), Armas-Cruz (2011), Ayuso (2006), Bagur-Femenias et al. (2016), Chou (2014), Dief \& Font (2012), Font et al. (2012), Fraj et al. (2015), Freitas \& Almeida (2010), Graci \& Dodds (2008), Hu et al. (2015), Kasim \& Dzakiria (2009), Lai et al (2012), Leonidou et al. (2015), López-Gamero et al. (2011), Mensah (2014a), Mishra (2016), Oreja-Rodríguez \& Armas-Cruz (2012), Pereira-Moliner et al. (2012), Pistorello et al. (2015), Salgado \& Colombo (2015), Shah (2011), Sharma (2009), Sousa \& Eusébio (2013).

Alonso-Almeida \& Rodríguez-Antón (2011), Alonso-Almeida et al. (2017), Armas-Cruz (2011), Ayuso (2006), Bagur-Femenias et al. (2016), Best \& Thapa (2013), Bicknell \& McManus (2006), Boemi \& Avdimiotis (2015), Carrenho et al. (2012), Chan \& Wong (2006), Chan et al. (2009), Chou (2014), Chung \& Parker (2008), Cortijo (2003), Dief \& Font (2012), Dodds \& Holmes (2011), Duglio \& Beltramo (2016), Filimonau et al (2011), Font et al. (2012), Fraj et al. (2015), Freitas \& Almeida (2010), Garcia-Pozo et al. (2014), Graci \& Dodds (2008), Hu et al. (2015), Karatzoglou \& Spilanis (2010), Kasim \& Dzakiria (2009), Lai et al (2012), Lee (2010), Legrand et al. (2012), Leonidou et al. (2015), López-Gamero et al. (2011), Medeiros et al. (2012), Mensah (2014a, 2014b), Mishra (2016), Molina-Azorin (2015), Molina-Azorín et al. (2009), Oreja-Rodríguez \& Armas-Cruz (2012), Park \& Kim (2014), Pereira-Moliner et al. (2012), Pistorello et al. (2015), Rabontu \& Babucea (2015), Reynolds (2013), Rivera (2004, 2002), Rivera \& De Leon (2005), Salgado \& Colombo (2015), Sánchez-Medina et al. (2016), Sanchez-Ollero et al. (2012), Santos et al. (2017), Shah (2011), Sharma (2009), Sousa \& Eusébio (2013), Sozer (2010), Styles et al. (2015), Tang et al. (2014), Trung \& Kumar (2005), Warnken et al. (2005, 2004), Xuchão et al. (2010), Zubair et al (2011).

Armas-Cruz (2011), Best \& Thapa (2013), Carrenho et al. (2012), Chan et al. (2009), Chen \& Huang (2015), Chou (2014), Cortijo (2003).Dief \& Font (2012), Filimonau et al. (2011), Font et al. (2012), Freitas \& Almeida (2010), Georgei \& Bombeck (2012), Hu et al. (2015), Karatzoglou \& Spilanis (2010), Lai et al (2012), Le et al. (2006), Lee (2010), Mensah (2014a), Mishra (2016), Molina-Azorín et al. (2009), Oreja-Rodríguez \& Armas-Cruz (2012), Rabontu \& Babucea (2015), Salgado \& Colombo (2015), Sánchez-Medina et al. (2016), Santos et al. (2017), Shah (2011), Sousa \& Eusébio (2013), Styles et al. (2015), Tortella \& Tirado (2011), Trung \& Kumar (2005), Warnken et al. (2005), Zubair et al (2011). 


\section{Emissões atmosféricas:}

Emissões de $\mathrm{CO} 2$ e outros gases de efeito estufa associados à produção e consumo de energia térmica e elétrica no meio de hospedagem
Bardhan (2012), Chung \& Parker (2008), Cumo et al., (2015), Duglio \& Beltramo (2016), Fazelpour et al. (2014), Filimonau et al. (2011), Freitas \& Almeida (2010), Georgei \& Bombeck (2012), Hu et al., (2015), Karatzoglou \& Spilanis (2010), Kasim \& Dzakiria (2009), Lai et al (2012), Le et al. (2006), Legrand et al. (2012), López-Gamero et al. (2011), Mensah (2014a), Oreja-Rodríguez \& Armas-Cruz (2012), Rivera (2004), Santos et al., (2017), Sousa \& Eusébio (2013), Sozer (2010), Trung \& Kumar (2005), Warnken et al. (2004), Xuchão et al. (2010).

Armas-Cruz (2011), Le et al. (2006), Lee (2010), Leonidou et al. (2015), López-Gamero et al. (2011), Mensah (2014b), Mishra (2016), Molina-Azorin (2015), Oreja-Rodríguez \& Armas-Cruz (2012), Qi et al., (2017), Santos et al. (2017), Sousa \& Eusébio (2013), Trung \& Kumar (2005), Zubair et al (2011).

Qi et al., (2017), Santos et al. (2017).

Não especificado

FONTE: elaboração própria

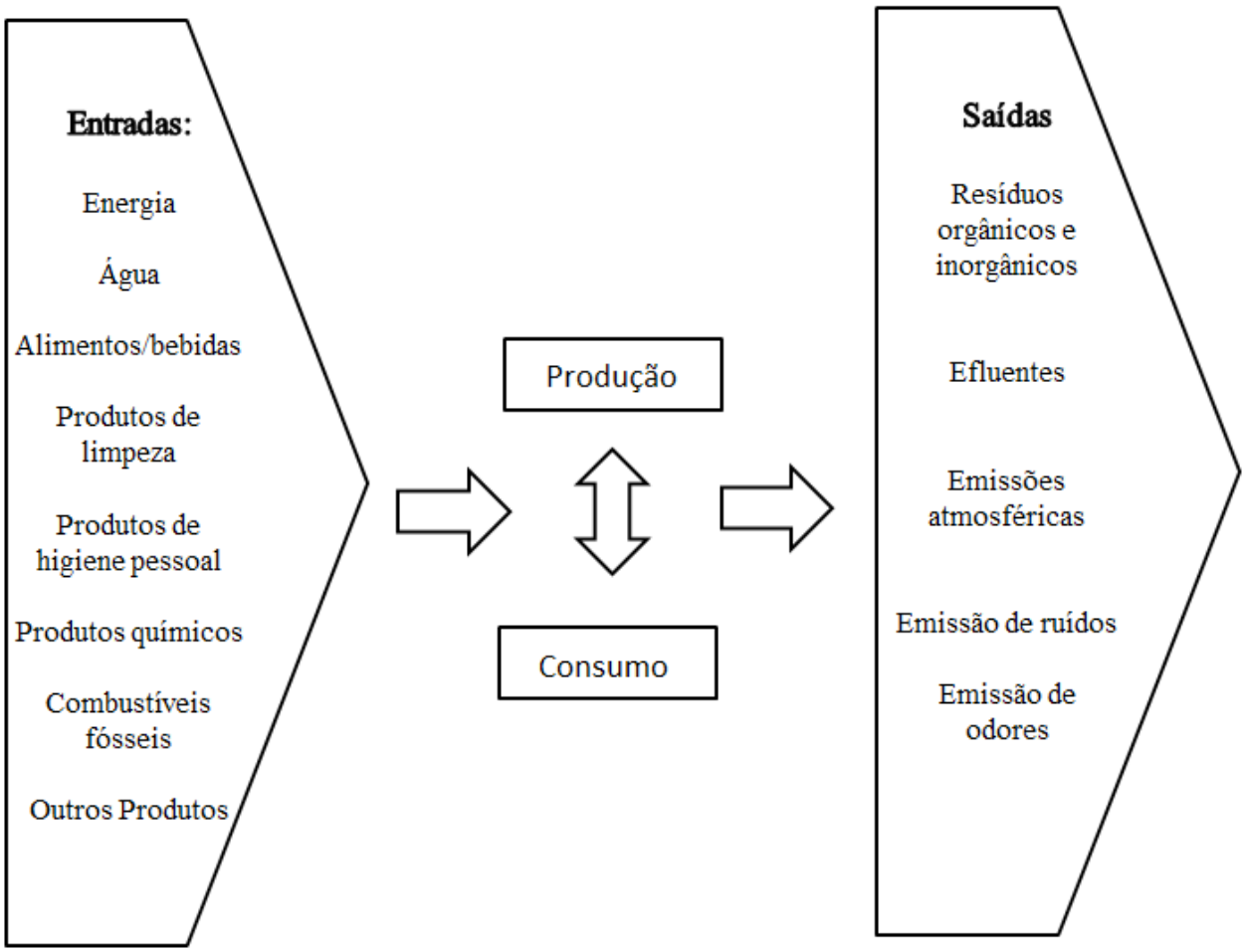

FIGURA 5 - Conceito do ciclo de vida adaptado aos serviços de hospedagem (etapas de produção e uso do produto).

FONTE: Adaptado de ABNT ISO Guia 64 (2010). 


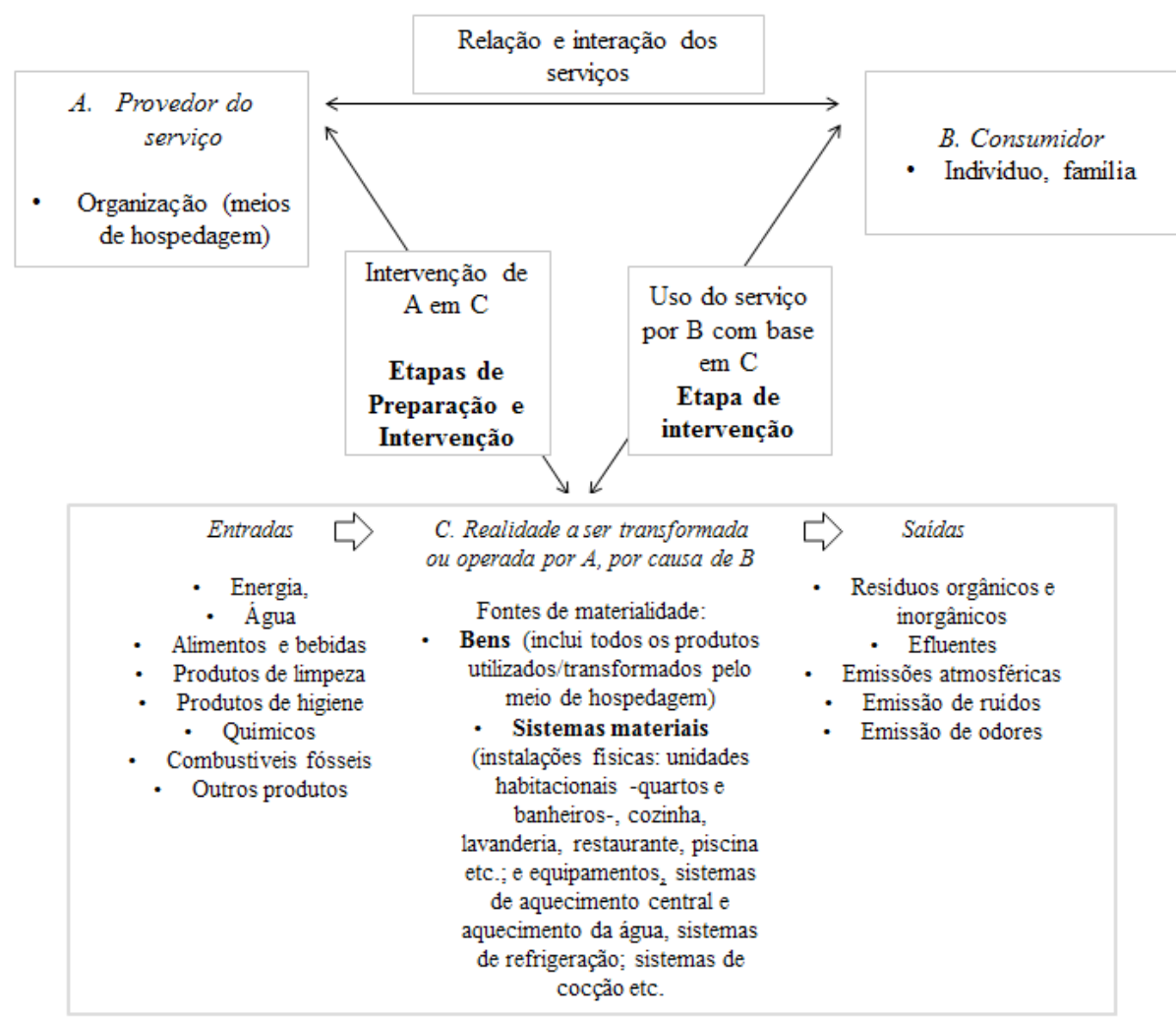

FIGURA 6 - Triângulo dos serviços de hospedagem ampliado para a incorporação dos aspectos ambientais associados às fontes de materialidade do serviço nas etapas de produção e uso.

FONTE: Elaboração própria com base em Gadrey (2002); Fourcroy et al (2015); Djellal \& Gallouj (2016).

1) Intervenção: é uma combinação de diferentes operações de acordo com a natureza do seu suporte ou objetivo (Gadrey, 1991). Essas operações podem ser materiais (transformação, manutenção ou transporte de bens), informacionais (produção, circulação, arquivamento ou tratamento de informações codificadas), cognitivas (processamento de conhecimento) e relacionais (serviço apoiado no próprio cliente);
2) Preparação: refere-se à preparação dos locais onde a intervenção do serviço ocorre (limpeza, calefação etc.) e dos prestadores de serviço (formação, recepção etc.). Essa preparação pode se prolongar durante a intervenção também (calefação e iluminação do local, por exemplo);

3) Deslocamento dos consumidores e dos prestadores de serviço: Pode se tratar do deslocamento do cliente/usuário em direção ao prestador do serviço (como é o caso dos meios de hospedagem, 
por exemplo), do prestador de serviço em direção ao cliente (serviços a domicílio, por exemplo) ou de ambos simultaneamente (transporte de pessoas, por exemplo).

Nessa pesquisa são considerados os estágios de produção e uso do ciclo de vida dos serviços de hospedagem. Tais estágios estão implícitos nas prestações elementares, preparação e intervenção realizadas nos meios de hospedagem. Sendo assim, a preparação é associada aos aspectos ambientais (entradas e saídas) referentes, por exemplo, à limpeza das instalações, climatização do ambiente, disponibilização de produtos de higiene, manutenção das piscinas e jardins e demais atividades de preparação dos meios de hospedagem como um todo para a recepção dos hóspedes. E a intervenção engloba uma combinação de diversas operações, como, por exemplo: reservas (operação informacional), uso de tecnologias da informação e comunicação (operação informacional), preparação de alimentos nos restaurantes dos meios de hospedagem (operação material), consumo de papel na recepção (operação material), e o acolhimento dos hóspedes de modo geral (operação relacional).

As fontes de materialidade estão no centro da identificação dos aspectos ambientais das atividades de serviços, conforme ilustrado na figura 4 e são classificadas em:

- Bens utilizados e/ou transformados para atender a demanda dos hóspedes, tais como: alimentos, bebidas, produtos de limpeza, produtos de higiene pessoal, produtos químicos;

- Sistemas materiais: a) instalações físicas (unidades habitacionais - quartos, banheiros, suítes -, restaurante, piscina, lavanderia etc.); b) equipamentos, sistemas de aquecimento, sistemas de refrigeração, sistemas de cocção de alimentos etc.
Por fim, os serviços de hospedagem podem ser definidos como uma prestação de serviços realizada por uma organização (o meio de hospedagem) e exige a operação de instalações físicas (local onde o serviço é prestado), a partir da demanda do hóspede, a utilização e/ou transformação de bens e a utilização de sistemas materiais necessários para prover os serviços.

\section{Considerações finais}

O debate atual sobre serviços é marcado pelo questionamento do argumento teórico da imaterialidade dos serviços em oposição à materialidade dos bens. Para o propósito deste artigo, a discussão da materialidade dos serviços é relevante ao contribuir para esclarecer a interação entre serviços e meio ambiente, com foco nos serviços de hospedagem. Análises que buscam avançar na compreensão da natureza dos serviços discutem e questionam o argumento teórico da imaterialidade intrínseca dos serviços em oposição à materialidade dos bens. O produto e o processo produtivo do serviço são considerados indissociáveis.

Assim, o objetivo da presente pesquisa, de discutir a materialidade dos serviços de hospedagem, envolve a identificação das fontes dessa materialidade.

Com base no modelo do triângulo de serviços, identifica-se que tais fontes de materialidade se manifestam na "realidade a ser transformada ou operada pelo provedor do serviço por causa do consumidor". E são geradoras de entradas e saídas, conforme entendimento do conceito do ciclo de vida. Esse conceito se refere à consideração de todos os aspectos ambientais de um produto (bens e serviços) em todos os estágios de seu ciclo de vida. 
A atividade de serviço requer entradas (recursos) e, consequentemente, gera saídas (liberações) que podem causar efeitos adversos ao meio ambiente. As entradas e saídas presentes no ciclo de vida do produto (bens e serviços) esclarecem os aspectos ambientais relevantes para a consideração sistemática de questões ambientais e para entender como o produto interage com o ambiente. Para os meios de hospedagem, a provisão dos serviços requer as seguintes entradas: água, energia, alimentos e bebidas, produtos de limpeza, produtos de higiene pessoal, produtos químicos, combustíveis fósseis e outros produtos. E, também, gera saídas: resíduos orgânicos e inorgânicos, efluentes e emissões atmosféricas, ruídos, odores.

O questionamento da suposta imaterialidade intrínseca dos serviços propõe o aprimoramento teórico sobre a natureza dos serviços. Tais avanços na fundamentação teórica podem contribuir para o entendimento e a análise da sustentabilidade no turismo. $\mathrm{O}$ foco deste artigo repousa na abordagem das fontes de materialidade necessárias para a provisão dos serviços de hospedagem. Nesse sentido, a ampliação da estrutura analítica do triângulo dos serviços proposta neste trabalho permite: considerar os estágios de produção e uso (preparação e intervenção) do ciclo de vida dos serviços em foco; e analisar as fontes de materialidade situando-as no centro da interação dos serviços de hospedagem com o meio ambiente (aspectos ambientais). Evidenciam-se as principais fontes de materialidade que se manifestam no suporte material (bens, instalações físicas, equipamentos) requerido na prestação dos serviços de hospedagem.

\section{Referências}

ABNT. ABNT ISO Guia 64: Guia para consideração de questões ambientais em normas de produtos. Rio de Janeiro, 2010.

Alonso-Almeida, M.; Rodríguez-Antón, J. M. Organisational behaviour and strategies in the adoption of certified management systems: an analysis of the Spanish hotel industry. Journal of Cleaner Production, 19(13), 1455-1463, 2011. doi: 10.1016/j.jclepro.2011.04.015

Alonso-Almeida, M.; Robin, C. F.; Pedroche, M. S. C.; Astorga, P. S. Revisiting green practices in the hotel industry: A comparison between mature and emerging destinations. Journal of Cleaner Production, 140, 1415-1428, 2017. doi: 10.1016/j.jclepro.2016.10.010

Armas-Cruz, Y. Determinants of environmental management and implications for firms' economic performance. Journal of Environmental Planning and Management, 54(8), 10771106, 2011. doi: 10.1080/09640568.2010.549401

Ayuso, S. Adoption of voluntary environmental tools for sustainable tourism: Analysing the experience of Spanish hotels. Corporate Social Responsibility and Environmental Management , 13(4), 207-220, 2006.doi : 10.1002/csr.103

Bagur-Femenias, L.; Celma, D.; Patau, J. The Adoption of Environmental Practices in Small Hotels. Voluntary or Mandatory? An Empirical Approach. Sustainability, 8(7), 695, 2016. doi: 10.3390/su8070695

Bardhan, S. Adopting environment responsive strategies for existing buildings: A prescriptive study. International Journal of Sustainable Development and Planning, 7 (3), 333-344, 2012. doi :10.2495/ECO110081

Beni, M. C. Sistema de turismo-SISTUR: estudo do turismo face à moderna teoria de sistemas. Revista Turismo em Análise, 1(1), 15-34, 1990. doi: 10.11606/issn.1984-4867. v1i1p15-34

Beni, M. C. Como Certificar o Turismo Sustentável?. Revista Turismo em Análise, 14(2), 5-16, 2003. doi: 10.11606/ issn.1984-4867.v14i2p5-16

Best, M. N.; Thapa, B. Motives, facilitators and constraints 
of environmental management in the Caribbean accommodations sector. Journal of Cleaner Production, 52, 165-175, 2013. doi: 10.1016/j.jclepro.2013.03.005

Bicknell, S.; Mcmanus, P. The canary in the coalmine: Australian ski resorts and their response to climate change. Geographical Research, 44(4), 386-400, 2006. 10.1111/j.1745-5871.2006.00409.x

Boemi, S. N.; Avdimiotis, S. Sustainability assessment in tourist facilities. Journal of environmental protection and ecology, 16(4), 1560-1564, 2015. Disponível em: < http:// www.jepe-journal.info/vol-16-no-4-2015>

Brasil. Lei n. 11.771, de 17 de setembro de 2008. Dispõe sobre a Política Nacional de Turismo, define as atribuições do Governo Federal no planejamento, desenvolvimento e estímulo ao setor turístico; revoga a Lei ${ }^{\circ} 6.505$, de 13 de dezembro de 1977, o Decreto-Lei $n^{\circ} 2.294$, de 21 de novembro de 1986, e dispositivos da Lei $\mathrm{n}^{\circ} 8.181$, de 28 de março de 1991; e dá outras providências. Diário Oficial da República Federativa do Brasil, Brasília, DF, set. 2008.

Buckley, R. Sustainable tourism: Research and reality. Annals of Tourism Research, 39(2), 528-546, 2012. doi: 10.1016/j.annals.2012.02.003

Carrenho, C. R.; de Figueiredo, R. S.; Sabino, J. Percepção de atores sociais quanto ao uso de tecnologias alternativas e mitigadoras de impacto ambiental por empreendimentos hoteleiros em Bonito, Mato Grosso do Sul. Revista Brasileira de Gestão e Desenvolvimento Regional, 8(2), 244-274, 2012. Disponível em: < www.rbgdr.net/revista/>

Chan, W.; Wong, K.; Lo, J. Hong Kong hotels' sewage: environmental cost and saving technique. Journal of Hospitality \& Tourism Research, 33(3), 329-346, 2009. doi: 10.1177/1096348009338525

Chan, E. S. W. Implementing environmental management systems in small-and medium-sized hotels: Obstacles. Journal of Hospitality \& Tourism Research, 35(1), 3-23, 2011. doi: 10.1177/1096348010370857

Chan, E. S. W.; Wong, S. C. K. Motivations for ISO 14001 in the hotel industry. Tourism Management, 27(3), 481-492, 2006. doi: 10.1016/j.tourman.2004.10.007

Chen, H.-S.; Huang, W.-S. Development Of An Ecological
Footprint For Accommodation Facilities In Taroko National Park, Taiwan. International Journal of Design \& Nature and Ecodynamics, 10(2), 165-173, 2015. doi: 10.2495/ DNE-V10-N2-165-173

Chou, C.-J. Hotels' environmental policies and employee personal environmental beliefs: Interactions and outcomes. Tourism Management, 40, 436-446, 2014. doi 10.1016/j.tourman.2013.08.001

Chung, L. H.; Parker, L. D. Integrating hotel environmental strategies with management control: a structuration approach. Business Strategy and the Environment, 17(4), 272-286, 2008.doi: $10.1002 /$ bse.546

Cortijo, P. Solar energy and eco-design in the tourism sector. Industry and Environment, 26 (2), 28, 2003. Disponível em: < http://www.uneptie.org/division/media/review/ vol26no2-3/voL26_no2-3.htm\#contents>.

Cumo, F.; Garcia, D. A.; Stefanini, V.; Tiberi, M. Technologies and strategies to design sustainable tourist accommodations in areas of high environmental value not connected to the electricity grid. International Journal of Sustainable Development and Planning, 10(1), 20-28, 2015. doi: 10.2495/SDP-V10-N1-20-28

Dief, M.; Font, X. Determinants of environmental management in the Red Sea Hotels: personal and organizational values and contextual variables. Journal of Hospitality \& Tourism Research, 36(1), 115-137, 2012. doi: $10.1177 / 1096348010388657$

Djellal, F.; Gallouj, F. The productivity challenge in services: measurement and strategic perspectives. The Service Industries Journal, 33(3-4), 282-299, 2013. doi: 10.1080/02642069.2013.747519

Djellal, F.; Gallouj, F. Green and sustainable innovation in a service economy. In: Bryson, J.; Daniels, P. (Eds.). The Handbook of Service Business: Management, Marketing, Innovation and Internationalization. Cheltenham: Edward Elgar, p. 83-104, 2015a.

Djellal, F.; Gallouj, F. Service innovation for sustainability: paths for greening through service innovation. XXV RESER conference "Service development and innovation for prosperity and human well-being in the 21 st century", Copenhagen, 2015b. 
Djellal, F.; Gallouj, F. Innovation de service et développement durable: quelles trajectoires pour un verdissement par l'innovation de service?. In: Journées Internationeles du Risque. Niort: Université de Poitiers, 2016.

Dodds, R.; Holmes, M. R. Sustainability in Canadian B\&Bs: comparing the east versus west. International Journal of Tourism Research, 13(5), 482-495, 2010. doi: 10.1002/ jtr.822

Duglio, S.; Beltramo, R. Environmental Management and Sustainable Labels in the Ski Industry: A Critical Review. Sustainability, 8(9), 851, 2016. doi: 10.3390/ su8090851

Echarri, V.; Aldea, F. J.; Gómez, J. G.; Del Hombrebueno, J. R. The Integrated Renovation Of High-rise Hotels On The Spanish Mediterranean Coast. International Journal of Sustainable Development and Planning, 12(2), 205-216, 2017. doi: 10.2495/SDP-V12-N2-205-216.

Fazelpour, F.; Soltani, N.; Rosen. M. A. Feasibility of satisfying electrical energy needs with hybrid systems for a medium-size hotel on Kish Island, Iran. Energy, 73, 856865, 2014. doi: 10.1016/j.energy.2014.06.097

Felix, V.; Santos, J. Proposta de uma metodologia de avaliação de desempenho ambiental para o setor hoteleiro. Revista Acadêmica Observatório de Inovação do Turismo, 7(4), 34-53, 2013. doi: 10.12660/oit.v7n4.11411

Filimonau, V.; Dickinson, J.; Robbins, D.; Huijbregts, M. A. Reviewing the carbon footprint analysis of hotels: Life Cycle Energy Analysis (LCEA) as a holistic method for carbon impact appraisal of tourist accommodation. Journal of Cleaner Production, 19 (17), 1917-1930, 2011.doi: 10.1016/j.jclepro.2011.07.002

Fisk, R. P.; Brown, S. W.; Bitner, M. J. Tracking the evolution of the services marketing literature. Journal of retailing, 69(1), 61-103, 1993. doi: 10.1016/s0022-4359(05)80004-1

Font, X.; Walmsley, A.; Cogotti, S.; McCombes, L.; Häusler, N. Corporate social responsibility: The disclosure-performance gap. Tourism Management, 33(6), 1544-1553, 2012. doi: 10.1016/j.tourman.2012.02.012

Fourcroy, C.; Gallouj, F.; Decellas, F. Energy consumption in service industries: challenging the myth of non-materiali- ty. Ecological Economics, 81, 155-164, 2012. doi: 10.1016/j. ecolecon.2012.07.003

Fourcroy, C.; Gallouj, F.; Decellas, F. La matérialité invisible des services et ses implications énergétiques: une estimation de l'énergie grise par la méthode input-output. Revue d'économie industrielle, 1, 43-72, 2015. doi: 10.4000/rei.6072̂

Fraj, E.; Matute, J.; Melero, I. Environmental strategies and organizational competitiveness in the hotel industry: The role of learning and innovation as determinants of environmental success. Tourism Management, 46, 30-42, 2015.doi : 10.1016/j.tourman.2014.05.009

Freitas, A. L. P.; Almeida, G. M. M. Avaliação do nível de consciência ambiental em meios de hospedagem: uma abordagem exploratória. Sociedade \& Natureza, 22(2), 405-417, 2010. doi: 10.1590/S1982-45132010000200013

Gadrey, J. Le service n'est pas un produit: quelques implications pour l'analyse économique et pour la gestion. Politiques et Management Public, 9(1), 1-24, 1991. doi: 10.3406/ pomap.1991.2973

Gadrey, J. The characterization of goods and services: an alternative approach. Review of Income and Wealth, 46(3), 369-387, 2000. doi: 10.1111/j.1475-4991.2000.tb00848.x

Gadrey, J. The misuse of productivity concepts in services: lessons from a comparison between France and the United States. In: Gadrey, J.; Gallouj, F (Eds). Productivity, Innovation and Knowledge in Services: New Economic and Socio-economic Approaches. Cheltenham: Edward Elgar, p. $26-53,2002$.

Gadrey, J. The environmental crisis and the economics of services: the need for revolution. In: Gallouj F.; Djellal F. (Eds.). The Handbook of Innovation and Services: A Multi-disciplinary Perspective. Cheltenham: Edward Elgar, 93-125, 2010.

Gallouj, F.; Savona, M. Innovation in services: a review of the debate and a research agenda. Journal of evolutionary economics, 19(2), 149-172, 2009. doi: 10.1007/s00191008-0126-4

Garcia-Pozo, A.; Sanchez-Ollero, J. L.; Marchante-Mera, A. Environmental good practices, quality certifications and 
productivity in the Andalusian hotel sector. International Journal of Environmental Research, 8(4), 1185-1194, 2014. doi: 10.22059/IJER.2014.811

Georgei, M.; Bombeck, H. Energy Use In Sharm El-sheikh Resort In Egypt. International Journal of Sustainable Development and Planning, 7(4), 412-427, 2012. doi: 10.2495/ SDP-V7-N4-412-427

Gil, A. C. Métodos e técnicas de pesquisa social. São Paulo: Atlas, 1991

Graci, S.; Dodds, R. Why go green? The business case for environmental commitment in the Canadian hotel industry. Anatolia, 19(2), 251-270, 2008. doi: 10.1080/13032917.2008.9687072

GSTC. GSTC History. Disponível em: <https://www. gstcouncil.org/about/about-us/>. Acesso em: maio 2018.

GSTC. GSTC Hotel Criteria: Suggested Performance Indicators Preamble. Washington DC, 12 f, 2016.

Gummesson, E. The future of service is long overdue. In: Maglio, P.; Kieliszewski, C.; Spohrer,J. The Handbook of service science. New York: Springer, 625-642, 2010.

Hill, P. Tangibles, intangibles and services: a new taxonomy for the classification of output. The Canadian journal of economics, 32(2), 426-446, 1999. doi: 10.2307/136430

Hu, A. H.; Huang, C. Y.; Chen, C. F.; Kuo, C. H.; Hsu, C. W. Assessing carbon footprint in the life cycle of accommodation services: the case of an international tourist hotel. International Journal of Sustainable Development \& World Ecology, 22(4), 313-323, 2015. doi: 10.1080/13504509.2015.1049674

IPEA. Perfil da mão de obra do turismo no Brasil nas atividades características do turismo e em ocupações. Brasília, 66f, 2014.

Karatzoglou, B.; Spilanis, I. Sustainable tourism in Greek islands: the integration of activity-based environmental management with a destination environmental scorecard based on the adaptive resource management paradigm. $\mathrm{Bu}$ siness Strategy and the Environment, 19(1), 26-38, 2010. doi: $10.1002 /$ bse. 627

Kasim, A.; Dzakiria, H. Encouraging environmental management among small and medium accommodations (SMAs) through e-learning initiative. Turkish Online Journal of Distance Education, 10(2), 162-174, 2009. Disponível em: < http://dergipark.ulakbim.gov.tr/tojde/article/ view $/ 5000102587 / 5000095684>$

Kon, A. Características e funções dos serviços. In: Kon, A. Economia de Serviços: teoria e evolução no Brasil. Rio de Janeiro: Elsevier, 47-62, 2004.

Körössy, N. Do turismo predatório ao turismo sustentável: uma revisão sobre a origem e a consolidação do discurso da sustentabilidade na atividade turística. $\mathrm{Ca}$ derno Virtual de Turismo, 8(2), 2008. Disponível em: http://www.ivt.coppe.ufrj.br/caderno/index.php/caderno/ article/view/238

Lai, J. H.; Yik, F. W.; Man, C. S. Carbon audit: a literature review and an empirical study on a hotel. Facilities, 30 (9/10), 417-431, 2012. doi: 10.1108/02632771211235233

Le, Y. et al. Environmental management: A study of Vietnamese hotels. Annals of Tourism Research, 33(2), 545-567. 2006. doi: 10.1016/j.annals.2006.01.002

Lee, O. A. Coastal resort development in Malaysia: A review of policy use in the pre-construction and post-construction phase. Ocean \& Coastal Management, 53(8), 439-446, 2010. doi: 10.1016/j.ocecoaman.2010.06.010

Lee, T. H.; Hsieh, H. Indicators of sustainable tourism: A case study from a Taiwan's wetland. Ecological Indicators, 67, 779-787, 2016. doi: 10.1016/j.ecolind.2016.03.023

Legrand, W.; Kirsche, C.; Sloan, P.; Simons-Kaufmann, C. Making 202020 Happen: Is The Hospitality Industry Mitigating Its Environmental Impacts? The Barriers And Motivators That German Hoteliers Have To Invest In Sustainable Management Strategies And Technologies And Their Perceptions Of Online Self Help. WIT Transactions on Ecology and the Environment, 161, 115-126, 2012. doi: 10.2495/ST120101

Legrand, W.; Sloan, P.; Chen, J. S. Sustainability in the Hospitality Industry: Principles of sustainable operations. New York: Routledge, 378p., 2013.

Leonidou, L. C.; Leonidou, C. N.; Fotiadis, T. A.; Aykol, B. Dynamic capabilities driving an eco-based advantage and 
performance in global hotel chains: The moderating effect of international strategy. Tourism Management, 50, 268-280, 2015. doi: 10.1016/j.tourman.2015.03.005

Li, P.; Ryan, C.; Cave, J. Chinese rural tourism development: Transition in the case of Qiyunshan, Anhui.-2008-2015. Tourism Management, 55, 240-260, 2016. doi: 10.1016/j.tourman.2016.02.007

López-Gamero, M. D.; Molina-Azorín, J. F.; Claver-Cortes, E. The relationship between managers' environmental perceptions, environmental management and firm performance in Spanish hotels: a whole framework. International Journal of Tourism Research, 13(2), 141-163, 2011. doi: $10.1002 /$ jtr.805

Lovelock, C.; Gummesson, E. Whither Services Marketing. Search of a New Paradigm and Fresh Perspectives. Journal of Service Research, 7(1), 20-41, 2004. doi: $10.1177 / 1094670504266131$

Medeiros, M.; Machado, D. F. C.; Passador, J. L.; Passador, C. S. Adoção da certificação LEED em meios de hospedagem: esverdeando a hotelaria? Revista de Administração de Empresas, 52(2), 179-192, 2012. doi: 10.1590/S003475902012000200005 .

Mensah, I. Stakeholder pressure and hotel environmental performance in Accra, Ghana. Management of Environmental Quality: An International Journal, 25(2), 227-243, 2014a. doi: 10.1108/MEQ-01-2013-0009

Mensah, I. Different shades of green: Environmental management in hotels in Accra. International Journal of Tourism Research, 16(5), p. 450-461, 2014b. doi: 10.1002/jtr.1939

Mishra, P. P. How Green are Our Hotels? Evidence from Thailand. Environment and Urbanization ASIA, 7(1), 2016, p. 132-148, 2016. doi: 10.1177/0975425315619050

Mohonk Agreement. Mohonk Agreement: Proposal for an International Certification Program for Sustainable Tourism and Ecotourism, 2000.

Molina-Azorín, J. F.; Claver-Cortés, E.; Pereira-Moliner, J.; Tarí, J. J. Environmental practices and firm performance: an empirical analysis in the Spanish hotel industry. Journal of Cleaner Production, 17(5), 516-524, 2009. doi: 10.1016/j. jclepro.2008.09.001
Molina-Azorín, J. F.; Tarí, J. J.; Pereira-Moliner, J.; Lopez-Gamero, M. D.; Pertusa-Ortega, E. M. The effects of quality and environmental management on competitive advantage: A mixed methods study in the hotel industry. Tourism Management, 50, 41-54, 2015. doi: 10.1016/j. tourman.2015.01.008

Moss, S. E.; Ryan, C.; Wagoner, C. B. An empirical test of butler's resort product life cycle: Forecasting casino winnings. Journal of Travel Research, 41(4), 393-399, 2003. doi: $10.1177 / 0047287503041004008$

MTUR. Programa de Regionalização do Turismo - Roteiros do Brasil: Turismo e Sustentabilidade. Brasília, 126 f, 2007.

OMT. Indicators of Sustainable Development for Tourism Destinations: A Guidebook. Madrid, 516 f, 2004.

OMT. Understanding Tourism: Basic Glossary. Madrid, $4 \mathrm{f}, 2008$.

OMT. Why Tourism?. Disponível em: $<$ http://www2.unwto. org/content/why-tourism>. Acesso em: maio 2018.

Oreja-Rodríguez, J. R.; Armas-Cruz, Y. Environmental performance in the hotel sector: the case of the Western Canary Islands. Journal of Cleaner Production, 29, 64-72, 2012.doi: 10.1016/j.jclepro.2012.02.012

Park, J.; Kim, H. J.; Mccleary, K. W. The impact of top management's environmental attitudes on hotel companies' environmental management. Journal of Hospitality \& Tourism Research, 38(1), 95-115, 2014.doi: $10.1177 / 1096348012452666$

Pereira-Moliner, J.; Claver-Cortés, E.; Molina-Azorín, J. F.; Tarí, J. J. Quality management, environmental management and firm performance: direct and mediating effects in the hotel industry. Journal of Cleaner Production, 37, 82-92, 2012. doi : 10.1016/j.jclepro.2012.06.010

Peres Jr, M. R.; De Rezende, D. C. Gestão da sustentabilidade no segmento hoteleiro: estudo dos meios de hospedagem de Monte Verde, MG. Caderno Virtual de Turismo, 11 (2), 2011. Disponível em http://www.ivt.coppe.ufrj.br/caderno/ index.php/caderno/article/view/659

Pistorello, J.; De Conto, S. M.; Zaro, M. Geração de resíduos sólidos em um restaurante de um Hotel da Serra Gaúcha, Rio Grande do Sul, Brasil. Engenharia Sanitária 
e Ambiental, 20(3), 337-346, 2015. doi: 10.1590/S141341522015020000133231.

PNUMA; OMT. Making Tourism More Sustainable: A Guide for Policy Makers. Madrid: World Tourism Organization Publications, 2005.

Qi, M.; Li, X.; Zhu, E.; Shi, Y. Evaluation of perceived indoor environmental quality of five-star hotels in China: An application of online review analysis. Building and Environment, 111, 1-9, 2017. doi: 10.1016/j.buildenv.2016.09.027

Rabontu, C. I.; Babucea, A. G. The eco-labelling in tourism: energy efficiency way. Progress in Industrial Ecology: an International Journal, 9(1), 60-68, 2015. doi: 10.1504/ PIE.2015.069841

Rainforest Alliance. Guia de Boas Práticas para o Turismo Sustentável. Rainforest Alliance, 136 f, s/d.

Rattan, J. K. Is certification the answer to creating a more sustainable volunteer tourism sector?. Worldwide Hospitality and Tourism Themes, 7(2), 107-126, 2015. doi: 10.1108/ WHATT-12-2014-0047

Reynolds, P. Hotel companies and corporate environmentalism. Tourism \& Management Studies, 9(1), 7-12, 2013.

Rivera, J. Assessing a voluntary environmental initiative in the developing world: The Costa Rican Certification for Sustainable Tourism. Political Science, 35(4), 333-360, 2002. doi: 10.1023/A:1021371011105

Rivera, J. Institutional pressures and voluntary environmental behavior in developing countries: Evidence from the Costa Rican hotel industry. Society \& Natural Resources, 17(9), 779-797, 2004. doi: 10.1080/08941920490493783

Rivera, J.; De Leon, P. Chief executive officers and voluntary environmental performance: Costa Rica's certification for sustainable tourism. Political Science, 38(2-3), 107-127, 2005. doi: 10.1007/s11077-005-6590-x

Salgado, C. C. R.; Colombo, C. R. Sistema de Gestão Ambiental no Verdegreen Hotel-João Pessoa/PB: Um Estudo de Caso Sob a Perspectiva da Resource-Based View. Revista de Administração Mackenzie, 16(5), 195-225, 2015. doi: 10.1590/1678-69712015/administracao.v16n5p195-225

Sánchez-Medina, P. S.; Díaz-Pichardo, R.; Cruz-Bautista,
M. Stakeholder Influence on the Implementation of Environmental Management Practices in the Hotel Industry. International Journal of Tourism Research, 18(4), 387-398, 2016. doi: 10.1002/jtr.2056

Sánchez-Ollero, J. L.; García-Pozo, A.; Marchante-Lara, M. Environment and Strategic Behaviour: The Case of Hotelsin and Alusia (SPAIN). International Journal of Environmental Research, 6(4), 1067-1076, 2012. doi : 10.22059/IJER.2012.577

Santos, R. A.; Méxas, M. P.; Meiriño, M. J. Sustainability and hotel business: criteria for holistic, integrated and participative development. Journal of Cleaner Production, 142, 217-224, 2017. doi: 10.1016/j.jclepro.2016.04.098

Sealey, K. S.; Smith, J. Recycling for small island tourism developments: Food waste composting at Sandals Emerald Bay, Exuma, Bahamas. Resources, Conservation and Recycling, 92, 25-37, 2014. doi: 10.1016/j.resconrec.2014.08.008

Shah, K. U. Strategic organizational drivers of corporate environmental responsibility in the Caribbean hotel industry. Political Science, 44(4), 321-344, 2011. doi: 10.1007/ s11077-011-9130-x

Sharma, S. The mediating effect of information availability between organization design variables and environmental practices in the Canadian hotel industry. Business Strategy and the Environment, 18(4), 266-276, 2009. doi : 10.1002/ bse. 603

Sousa, N.; Eusébio, C. Análise da gestão ambiental nos hotéis portugueses. Rev. Portuguesa e Brasileira de Gestão, 12(2), 60-74, 2013. Disponível em: <http:// www.scielo.mec.pt/scielo.php?script=sci_arttext\&pi$\mathrm{d}=\mathrm{S} 1645-44642013000200006>$

Sousa, P. G. D.; Matias, E.; Selva, V. S. From residential tourism to tourist real estate complexes: the appropriation of the coastal zone in the northeast of brazil by tourist real estate activities. Ambiente \& Sociedade, 19(3), 177-198, 2016. doi: 10.1590/1809-4422ASOC141673V1932016

Sozer, H. Improving energy efficiency through the design of the building envelope. Building and Environment, 45(12), 2581-2593, 2010. doi: 10.1016/j.buildenv.2010.05.004 
Styles, D.; Schoenberger, H.; Galvez-Martos, J. L. Water management in the European hospitality sector: Best practice, performance benchmarks and improvement potential. Tourism Management, 46, 187-202, 2015. doi: 10.1016/j.tourman.2014.07.005

Tang, Y. H.; Amran, A.; Goh, Y. N. Environmental management practices of hotels in Malaysia: stakeholder perspective. International Journal of Tourism Research, 16(6), 586-595, 2014. doi: 10.1002/jtr.1952

Tether, B.; Metcalfe, J. S. Services and systems of Innovation. In: Malerba, F. (Ed.). Sectoral systems of innovation: concepts, issues and analyses of six major sectors in Europe. Cambridge: Cambridge University Press, 287-322, 2004.

Tortella, B. D.; Tirado, D. Hotel water consumption at a seasonal mass tourist destination. The case of the island of Mallorca. Journal of Environmental Management, 92(10), 2568-2579, 2011. doi: 10.1016/j.jenvman.2011.05.024

Torres, Y. D.; Nodal, Y. V.; Yanes, J. P. M.; Torres, Y. M. Feasibility evaluation of two solar cooling systems applied to a cuban hotel. Comparative analysis. Ingeniería Energética, 37(1), 35-44, 2016.

Trung, D. N.; Kumar, S. Resource use and waste management in Vietnam hotel industry. Journal of Cleaner Production, 13(2), 109-116, 2005. doi: 10.1016/j.jclepro.2003.12.014

UNESCO; PNUMA. Sustainable Tourism Development in UNESCO Designated Sites in South-Eastern Europe. Bonn: ETE, $43 \mathrm{f}$, s/d.
Warnken, J.; Bradley, M.; Guilding, C. Exploring methods and practicalities of conducting sector-wide energy consumption accounting in the tourist accommodation industry. Ecological Economics, 48(1), 125-141, 2004. doi 10.1016/j.ecolecon.2003.08.007

Warnken, J.; Bradley, M.; Guilding, C. Eco-resorts vs. mainstream accommodation providers: an investigation of the viability of benchmarking environmental performance. Tourism Management, 26(3), 367-379, 2005. doi: 10.1016/j.tourman.2003.11.017

Weiermair, K.; Peters, M.; Schuckert, M. Destination development and the tourist life-cycle: implications for entrepreneurship in Alpine tourism. Tourism Recreation Research, 32(1), 83-93, 2007. doi: 10.1080/02508281.2007.11081526

WTTC. Travel \& Tourism: Economic Impact 2017 Brazil. World Travel and Tourism Council, 24 f, 2017.

Xuchao, W.; Priyadarsini, R.; Eang, L. S. Benchmarking energy use and greenhouse gas emissions in Singapore's hotel industry. Energy Policy, 38(8), 4520-4527, 2010. doi : 10.1016/j.enpol.2010.04.006

Young, C. E. F. Potencial de crescimento da economia verde no Brasil. Política Ambiental: Economia Verde: Desafios e Oportunidades, 8, 88-97, 2011. Disponível em: http:// www.ie.ufrj.br/images/gema/Gema_Artigos/2011/PoliticaAmbiental08portugues.pdf

Zubair, S.; Bowen, D.; Elwin, J. Not quite paradise: Inadequacies of environmental impact assessment in the Maldives. Tourism Management, 32(2), 225-234, 2011. doi: 10.1016/j.tourman.2009.12.007 